\title{
Device-Related Complications Associated with Magec Rod Usage for Distraction-Based Correction of Scoliosis
}

\author{
Aakash Agarwal $^{1)}$, Amey Kelkar ${ }^{1)}$, Ashish Garg Agarwal' ${ }^{2}$, Daksh Jayaswal ${ }^{1)}$, Arvind Jayaswal $^{2)}$ and Vithal Shendge ${ }^{1)}$ \\ 1) Department of Bioengineering and Orthopaedics Surgery, University of Toledo, Toledo, USA \\ 2) Department of Orthopedics, Primus Super Speciality Hospital, New Delhi, India
}

\begin{abstract}
:
Introduction: Recent literature identifies similar failure rates such as anchor pull-out and rod breakage, but a higher unplanned revision surgery with MAGEC rods than with traditional growth rods. Besides known failure modes such as rod fracture, infection, etc., failure to noninvasively distract the rods was cited as the main cause of such unplanned surgeries. The source of these data ranges from multicenter cohort studies to singular case series. These studies included explanted implants that had undergone failure in distraction mechanism, rod fracture, or infection, or had reached their maximum length. Nevertheless, in addition to identifying the overall mode of failure, it is equally important to identify the large-scale incidence of exclusive failures in comparison with standard instrumentation failure modes in spine surgery.

Methods: The US Food and Drug Administration (FDA) Manufacturer and User Facility Device Experience (MAUDE) databases were searched for reports on MAGEC rods, and on standard instrumentation used for spinal fusion. The adverse events were recorded, tabulated, and analyzed.

Results: A search of the US FDA MAUDE database yielded reports of 163 device-related adverse events. These included distraction mechanism failure $(n=129)$, rod fracture $(n=24)$, and minor voluntary reports of infection and tissue discoloration $(n=10)$. For standard instrumentation usage in spine surgery, pedicle screw breakage post surgery $(n=336)$, set screw damage during surgery $(n=257)$, rod breakage post surgery $(n=175)$, interbody cage breakage during surgery $(n=118)$, and pedicle screw breakage during surgery $(n=75)$ were identified as the top 5 failure modes.

Conclusions: The study identified the distraction mechanism failure as the most common and growing complication associated with MAGEC rod usage in children with scoliosis, leading to unplanned invasive revision surgeries.
\end{abstract}

Keywords:

MAGEC rods, traditional growth rods, early onset scoliosis, distraction, rod fracture, complications

Spine Surg Relat Res 2020; 4(2): 148-151

dx.doi.org/10.22603/ssrr.2019-0041

\section{Introduction}

The noninvasive distraction mechanism of MAGEC rods used to lengthen the spine in children with scoliosis have made traditional growth rods (an invasive distraction mechanism) a second choice, that is, used only for underprivileged patients or healthcare systems ${ }^{1,2}$. Recent literature identifies similar failure rates such as anchor pull-out (11.8\%) and rod breakage $(10.6 \%)$, but a higher unplanned revision surgery with MAGEC rods than with traditional growth rods ${ }^{3)}$. Besides known failure modes such as rod fracture, infection, etc., failure to noninvasively distract the rods (mostly due to breakage of actuator pin or reduced force generation) was cited as the main cause (11.7\%-50\%) of such unplanned surgeries $^{3,4}$. A common observation among these implants was the generation of titanium wear debris (leading to metallosis) due to off-axis loading ${ }^{4,5}$. This is further escalated by failure of radial bearings and corrosion of underlying components. The source of these data ranges from multicenter cohort studies to singular case series ${ }^{4-7)}$. These studies included explanted implants that had undergone failure in distraction mechanism, rod fracture, or infection, or had reached their maximum length. Nevertheless, in addition to identifying the overall mode of failure, it is equally important to identify the large-scale incidence reporting of exclusive failures in comparison with other standard failure

Corresponding author: Aakash Agarwal, Aakash.Agarwal@utoledo.edu

Received: May 23, 2019, Accepted: September 17, 2019, Advance Publication: October 20, 2019

Copyright (C) 2020 The Japanese Society for Spine Surgery and Related Research 


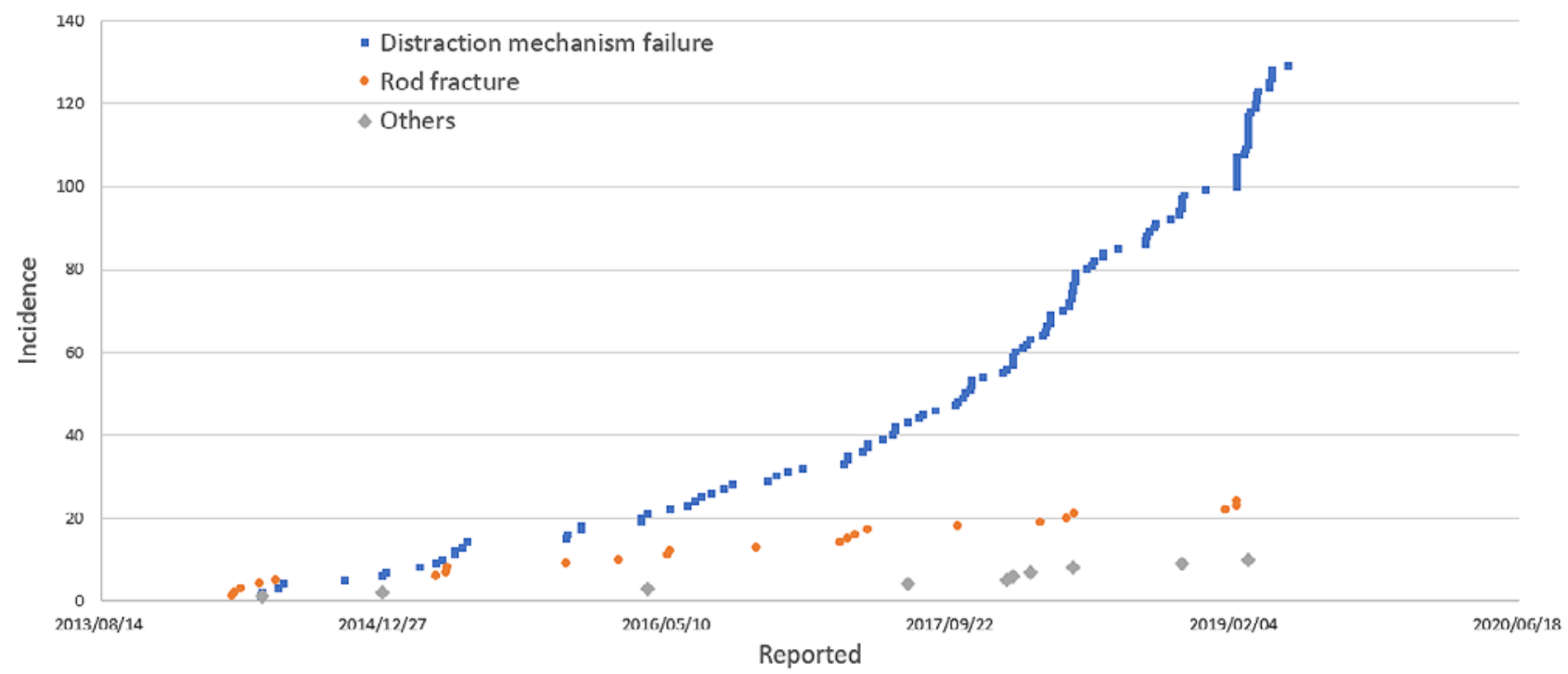

Figure 1. Adverse event reporting from the MAUDE database for MAGEC rod usage until June 2019.

modes in spine surgery. Therefore, the objective of the current study is to search the Manufacturer and User Facility Device Experience (MAUDE) database, which is a voluntary reporting system of adverse events involving medical devices since 1993, to identify the trend and frequency of adverse events related to the usage of MAGEC rods and standard instrumentation in spine surgery.

\section{Materials and Methods}

The United States Food and Drug Administration (FDA) of the Department of Human Services runs the MAUDE database. Adverse events are defined as potential and actual product use errors and product quality problems. The database contains information on medical devices that may have malfunctioned or caused a death or a serious injury. These adverse events are reported by the manufacturer or a healthcare professional (an operating room nurse or a surgeon). MAUDE may not include reports made according to exemptions, variances, or alternative reporting requirements granted under 21 Code of Federal Regulations 803.19. The FDA website was last accessed in June 2019, in preparation of data for this manuscript. The product code used was PGN (identifier for MAGEC rods), and all the categorial data based on event type were retrieved and analyzed to the date specified above. It is important to note that MAUDE data are not intended to be used either to evaluate absolute rates of adverse events (due to underreporting) or to compare adverse event occurrence rates between different devices (due to discrepancy in reporting between two devices). In addition to MAGEC rods, we also retrieved MAUDE data on the top 5 failure modes associated with standard instrumentation usage in spinal fusion; this does not include MAGEC rod failures, and may pertain to any possible diagnoses such as degenerative disc disease, spondylolisthesis, trauma, scoliosis, etc.

\section{Results}

A total of 163 incidences were recorded for MAGEC rodrelated adverse events, until June 2019. Of these, 129 events were failure of noninvasive distraction mechanism, 24 were for rod fracture, and 10 for other medical complications such as infection, tissue necrosis, wear particles, etc., (Fig. 1). The top 5 failure modes associated with standard instrumentation usage in spinal fusion included pedicle screw breakage post surgery $(n=336)$, set screw damage during surgery $(n=257)$, rod breakage post surgery $(n=175)$, interbody cage breakage during surgery $(n=118)$, and pedicle screw breakage during surgery $(n=75)$ (Fig. 2).

\section{Discussion}

The current study presents all the MAUDE reporting for adverse events related to the usage of MAGEC rods in growing children with scoliosis ${ }^{8)}$. Rod fracture is a known problem with all growth sparring systems; however, the failure to noninvasively distract the spine is exclusive to MAGEC rods only ${ }^{9,10}$. MAGEC rod's main benefit is that it allows for the noninvasive distraction of the growth rods. This benefit is realized by a drastic reduction in the number of consecutive surgeries, and in some cases, the possibility of reducing growth rod fracture and autofusion (caused due to gradual trauma to the spine via excessive distraction in traditional growth rod systems) ${ }^{11-16}$. However, the failure of this attribute, noninvasive distraction mechanism, reduces the overall efficacy of the device with newer studies even questioning if there is a real quality-of-life difference with use of MAGEC $\operatorname{rods}^{17)}$.

The results of the current study show that the most frequent clinical problem associated with the MAGEC rod is the failure of noninvasive distraction mechanism, which leads to invasive revision surgeries required to replace the device. These results also highlight the exponential increase 


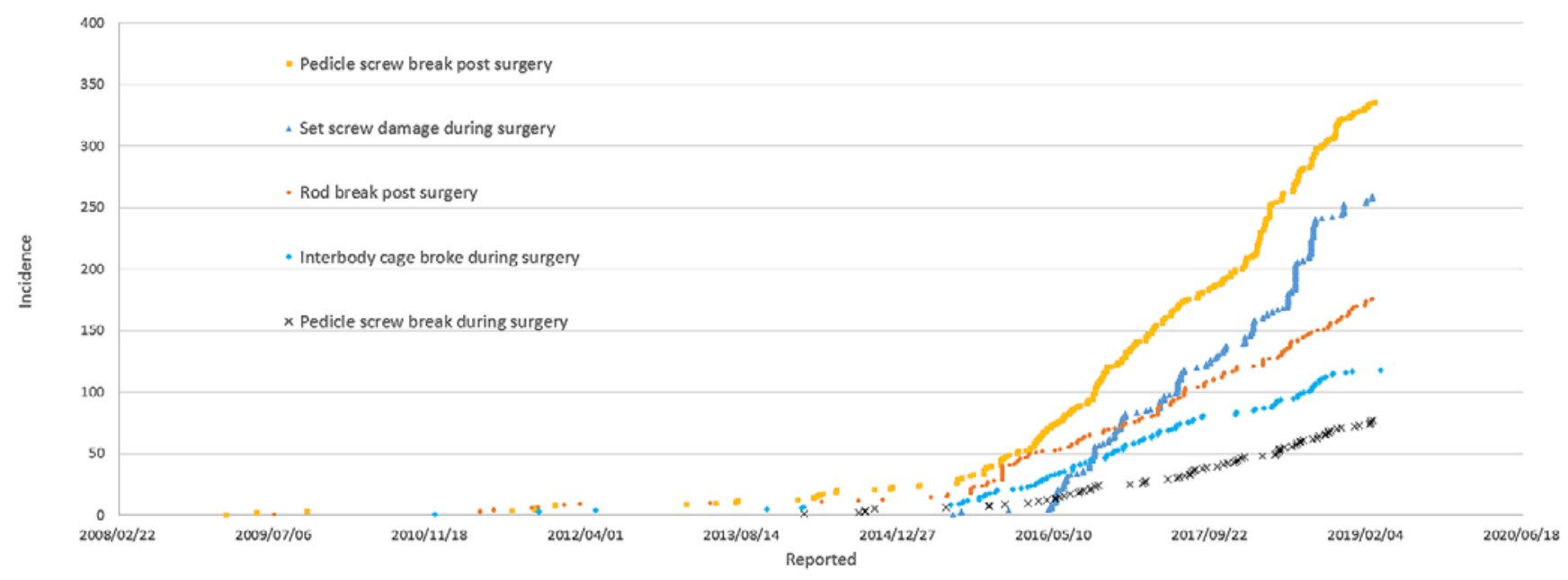

Figure 2. Adverse event reporting from the MAUDE database for the top 5 failure modes associated with standard instrumentation usage in spinal fusion until June 2019.

in such failure rates (Fig. 1). Furthermore, the MAUDE report on the top 5 failure modes associated with standard instrumentation usage in spinal fusion proves that such failures are substantially underreported.

Although the MAGEC rod has not completely replaced the traditional growth rod system, due to it unaffordability in underprivileged patients, it is still much more advantageous than its predecessor. The purpose of this study and many other clinical trials, or case series, is only to improve upon the existing noninvasive technology by thoroughly identifying the failure modes. In the case of MAGEC rods, it is the failure of its noninvasive distraction mechanism that needs attention. Further immediate research and development work should be undertaken to reduce these failure incidences in a clinical scenario. The limitation of the current study is that the patient demographics, surgical parameters, and the total number of surgeries performed (using MAGEC rods) in the United States within this period is unknown. The voluntary nature of such reporting also presents a challenge against excluding infrequently reported complications such as necrosis, which although is present in all cases (offaxis loading and wear), has only been reported 10 times in total. Despite these limitations, the type of adverse events and relatively high frequency of these events when compared to the top 5 failure modes associated with standard instrumentation usage in spinal fusion are a matter of great concern. In conclusion, the result of this study suggests a growing number of distraction mechanism failure associated with MAGEC rods. Better technical and clinical controls need to be set in place to avoid such adverse events, which leads to unplanned open surgeries. A higher distraction magnitude results in the generation of higher distraction forces, and this in combination with off-axis loading (exemplified by "growth marks") result in wear and breakage of MAGEC rod's components. Therefore, one method to reduce the propensity of such failures would be to apply minimum distraction at higher frequency, for example, $1.5-2.0 \mathrm{~mm}$ every month, instead of 4.5-6.0 mm every 3 months. This would also reduce tissue trauma and its effects, such as autofusion $^{11-16}$. Other technical methods to reduce attrition could include ceramic coating at wear generating surfaces, also applicable for growth guidance techniques ${ }^{18,20}$.

Conflicts of Interest: AA reports royalties from Paradigm Spine, Joimax, consultancy from Spinal Balance, and editorial board membership from Clinical Spine Surgery, Spine, outside the submitted work; the rest of the authors have nothing to disclose.

Author Contributions: Each co-author satisfied the 4 criteria as defined by ICMJE.

\section{References}

1. Jenks M, Craig J, Higgins J, et al. The MAGEC system for spinal lengthening in children with scoliosis: A NICE Medical Technology Guidance. Appl Health Econ Health Policy. 2014;12(6):58799.

2. Agarwal A, Goswami A, Vijayaraghavan GP, et al. Quantitative characteristics of consecutive lengthening episodes in Early-onset Scoliosis (EOS) patients with dual growth rods. Spine. 2019;44(6): 397-403.

3. Thakar C, Kieser DC, Mardare M, et al. Systematic review of the complications associated with magnetically controlled growing rods for the treatment of early onset scoliosis. Eur Spine J. 2018; 27(9):2062-71.

4. Joyce TJ, Smith SL, Rushton PR, et al. Analysis of explanted magnetically controlled growing rods from seven UK spinal centers. Spine. 2018;43(1):E16-22.

5. Teoh KH, Von Ruhland C, Evans SL, et al. Metallosis following implantation of magnetically controlled growing rods in the treatment of scoliosis: a case series. Bone Joint J. 2016;98(12):1662-7.

6. Teoh KH, Winson DM, James $\mathrm{SH}$, et al. Do magnetic growing rods have lower complication rates compared with conventional growing rods? Spine J. 2016;16(4):S40-4.

7. Tang N, Zhao H, Shen JX, et al. Magnetically controlled growing rod for early onset scoliosis: A systematic review and metaanalysis. World Neurosurg. 2019;125:e593-601.

8. Gurtcheff SE. Introduction to the MAUDE database. Clin Obstet 
Gynecol. 2008;51(1):120-3.

9. Teoh KH, Winson DM, James SH, et al. Magnetic controlled growing rods for early-onset scoliosis: a 4-year follow-up. Spine J. 2016;16(4):S34-9.

10. Yang JS, Sponseller PD, Thompson GH, et al, Growing Spine Study Group. Growing rod fractures: risk factors and opportunities for prevention. Spine. 2011;36(20):1639-44.

11. Cheung JP, Cahill P, Yaszay B, et al. Update on the magnetically controlled growing rod: tips and pitfalls. J Orthop Surg. 2015;23 (3):383-90.

12. Agarwal A, Agarwal AK, Jayaswal A, et al. Smaller interval distractions may reduce chances of growth rod breakage without impeding desired spinal growth: a finite element study. Spine Defor. 2014;2(6):430-6.

13. Cheung JP, Bow C, Samartzis D, et al. Frequent small distractions with a magnetically controlled growing rod for early-onset scoliosis and avoidance of the law of diminishing returns. J Orthop Surg. 2016;24(3):332-7.

14. Agarwal A, Agarwal AK, Jayaswal A, et al. Outcomes of optimal distraction forces and frequencies in growth rod surgery for different types of scoliotic curves: an in silico and in vitro study. Spine Defor. 2017;5(1):18-26.

15. Agarwal A, Jayaswal A, Goel VK, et al. Patient-specific distraction regimen to avoid growth-rod failure. Spine. 2018;43(4):E221-6.
16. Agarwal A, Jayaswal AK, Goel VK, et al. Letter to the Editor concerning "Rod fracture and lengthening intervals in traditional growing rods: is there a relationship?" by P. Hosseini et al. Eur Spine J (2016). doi:10.1007/s00586-016-4786-8. Eur Spine J. 2017;26(6):1696-7.

17. Bauer JM, Yorgova P, Neiss G, et al. Growing spine study group. early onset scoliosis: is there an improvement in quality of life with conversion from traditional growing rods to magnetically controlled growing rods? J Pediatr Orthop. 2019;39(4):e284-8.

18. Agarwal A, Aker L, Ahmad AA. Active apex correction with guided growth technique for controlling spinal deformity in growing children: A modified SHILLA technique. Glob Spine J. 2009.

19. Agarwal A, Aker L, Ahmad AA. Active apex correction (Modified SHILLA Technique) versus distraction-based growth rod fixation: What do the correction parameters say? Spine Surg Relat Res. 2019.

20. Lemans J, Kodigudla M, Kelkar A, et al. Spring distraction system for early onset scoliosis provides continuous distraction without a potential increase in rod fractures, compared to traditional growing rods. Spine Deform. 2018;6(6):819-20.

Spine Surgery and Related Research is an Open Access journal distributed under the Creative Commons Attribution-NonCommercial-NoDerivatives 4.0 International License. To view the details of this license, please visit (https://creativeco mmons.org/licenses/by-nc-nd/4.0/). 